\title{
Clinical Study of "Lingqi Huangban Granule" in Treating Choroidal Neovascularisation in Pathologic Myopia
}

\author{
Bingwen Lu${ }^{1}$, Xingwei $\mathrm{Wu}^{2}$, Lei Zhang1* \\ ${ }^{1}$ Ophthalmology Department, Seventh People's Hospital of Shanghai University of TCM, Shanghai, China \\ ${ }^{2}$ Ophthalmology Department, Shanghai General Hospital, Shanghai, China \\ Email:`704487389@qq.com
}

How to cite this paper: Lu, B.W., Wu, X.W. and Zhang, L. (2016) Clinical Study of "Lingqi Huangban Granule" in Treating Choroidal Neovascularisation in Pathologic Myopia. Chinese Medicine, 7, 125-132. http://dx.doi.org/10.4236/cm.2016.74013

Received: October 28, 2016

Accepted: November 17, 2016

Published: November 21, 2016

Copyright $\odot 2016$ by authors and Scientific Research Publishing Inc. This work is licensed under the Creative Commons Attribution International License (CC BY 4.0).

http://creativecommons.org/licenses/by/4.0/

\begin{abstract}
Objectives: To study the therapeutic effects of "Lingqi Huangban Granule" (LQHB) combined with intravitreal ranibizumab injection (IVR) for choroidal neovascularisation (CNV) in pathologic myopia (PM). Methods: This was a prospective, comparative, interventional study. Eighty eyes of eighty consecutive patients with myopic CNV were randomized into control group (IVR, 40 eyes) and integrative therapy group (IVR + LQHB, 40 eyes), each with a follow-up period of 12 months. IVR was given with an "on demand" regimen (Pro re nata, PRN). Final best corrected visual acuity (BCVA) and its change from baseline were the main outcome measures. Changes in optical coherence tomography (OCT) and central retinal thickness (CRT) were the secondary outcome measures. Results: BCVA of patients in the integrative group improved significantly (from $47.10 \pm 13.51$ letters to $90.72 \pm 12.98$ letter, $\mathrm{P}<0.05$ ), more than in the control group (from $42.90 \pm 13.18$ letters to 69.18 \pm 13.21 letters, $\mathrm{P}<0.05)$ since the third month after the initial treatment to the end of the follow-up. Mean OCT CRT reduced from $364.75 \pm 69.31$ to $278.93 \pm 33.05 \mathrm{um}$ $(\mathrm{P}<0.05)$ in the control group and from $370.73 \pm 65.31$ to $271.73 \pm 46.44 \mathrm{um}(\mathrm{P}<$ $0.05)$ in the integrative group, respectively. The mean number of intravitreal injections in the 1-year follow-up was also reduced. No ocular or systemic side effects were observed. Conclusion: LQHB combined with IVR therapy was an effective treatment for stabilizing and improving vision with fewer intravitreal injections. It appears to be an interesting option for this type of patient.
\end{abstract}

\section{Keywords}

Pathological Myopia, Choroidal Neovascularisation, "Lingqi Huangban Granule", Ranibizumab 


\section{Introduction}

Pathologic myopia (PM) has a high prevalence among Asian populations, especially among the younger age groups, and its prevalence has been reported to be increasing [1]. Myopic choroidal neovascularisation $(\mathrm{mCNV})$ is one of the most sight-threatening complications in highly myopic patients; their visual prognosis is poor if left untreated, and a high percentage develops visual loss [2]. Treatment options for patients with $\mathrm{mCNV}$ include laser photocoagulation, photodynamic therapy (PDT) and intravitreal injection of anti-VEGF drugs [3]. Since 2006, antiangiogenic drugs have become the first choice for this pathology because of their clinical efficacy and safety [4]. Nonetheless, repeated intravitreal injections are necessary to maintain vision improvement in a great number of cases [5]. This entails disadvantages as there is an increase in the potential risks of complications, discomfort for the patients, and health care expenses.

This study was carried out on $\mathrm{mCNV}$ patients with combined treatment integrating "Lingqi Huangban Granule" (LQHB) and intravitreal ranibizumab injection (IVR). The main objective was to determine if this association could achieve vision improvement for these patients at a 1-year follow-up, and at the same time decrease the number and frequency of intravitreal injections.

\section{Patients and Methods}

\subsection{Patients}

Eighty patients with central (sub-foveal and juxta-foveal) CNV were consecutively enrolled in this study. This prospective analysis was conducted at Ophthalmology Department of Shanghai Seventh People's Hospital from Jan. 2015 to Oct. 2016.

The study group comprised patients who met the inclusion criteria as follows: 1) highly myopic eyes, spherical equivalent greater than -6 diopters (D); 2) posterior pole myopic retinal changes (lacquer cracks, chorioretinal atrophy, papillary crescent, posterior staphyloma); 3) fluorescein angiography (FA) detection of sub-foveal or juxta-foveal active $\mathrm{CNV}$ on the basis of leakage and/or optical coherence tomographic (OCT) evidence of fluid; 4) Best corrected visual acuity (BCVA) > 20/400 at baseline; 5) duration of symptoms no longer than four weeks before enrollment; 6) minimum follow-up of 12 months, and 7) clear ocular media. Exclusion criteria included: 1) post inflammatory macular changes; 2) extra-foveal CNV; 3) age-related macular alterations; 4) refractive media opacities; 5) presence of other maculopathies as DR or RVO; 6) recent myocardial infarction or other thromboembolic events; 7) previous IV injections of any drugs; 8) ocular hypertension or glaucoma. Previous PDT if done more than 3 months prior to enrollment was not considered an exclusion criterion. Informed consent was obtained from patients before enrollment in the study. Ethical approval was obtained from Shanghai Seventh People's Hospital.

\subsection{Methods}

Eighty patients were randomly assigned to two groups using a randomized digital table: The integrative group (40 cases) and the control group (40 cases). The control group 
was treated with IVR alone. For the integrative group, besides IVR, patients also received LQHB (one pocket each time, 2 times daily). The treatment course was 1-year for both groups. Patients received an initial dose of $0.5 \mathrm{mg}(0.05 \mathrm{ml})$ of ranibizumab (Lucentis; Genentech) using the standard injection procedure in a surgical room. After the first intravitreal injection, ranibizumab was administered as "on demand" injections. Retreatment was considered according to angiographic, tomographic, or clinical signs of active lesion such as a loss of more than 1 line (5 letters) in BCVA and/or an increase of metamorphopsia, new retinal hemorrhage, evidence or increase of retin$\mathrm{al} /$ subretinal fluid or an increase in retinal thickness of at least $100 \mathrm{um}$ on OCT, or CNV leakage on FA images.

In addition to the treatment medicines, usages of any other traditional Chinese medicine or modern Western medicine were prohibited. A detailed history was taken to ascertain each patient's demographics and chief complaints, including duration of the symptom and presence of systemic diseases, such as hypertension, diabetes mellitus, cardiac diseases and hyperlipidemia. Each patient underwent BCVA measurement, biomicroscopy, tonometry and OCT (Zeiss, Germany) evaluation at baseline and every subsequent visit. Retinal FA (Heidelberg, Germany) was done at baseline within two weeks before the initial visit, then at intervals during the follow-up according to the ophthalmologist's judgement. BCVA was recorded using the Early Treatment Diabetic Retinopathy Study (ETDRS) chart at 4 meters distance using a log MAR scale. Results were recorded as $\operatorname{logMAR}$ values and the number of letters read. The primary outcome was considered to be the improvement of BCVA with a minimum of 5 letters (one ETDRS line) or the stabilization of visual acuity (VA). Secondary outcomes was the changes in OCT CRT.

\subsection{Statistical Analysis}

Test results were represented by $\mathrm{x} \pm \mathrm{s}$ using the SPSS 18.0 software. Descriptive statistics were used to summarize patient demographics and baseline ocular characteristics. Statistical methods such as t-test for independent samples, paired sample t-test and analysis of variance were used for testing and analysis. The Wilcox on test was used to compare the homogeneity between two related samples. In case of unrelated samples, the Mann-Whitney and Kruskal-Wallis tests were used, respectively. P values less than 0.05 were considered statistically significant in this study.

\section{Results}

\subsection{Patient Demographics and Baseline Characteristics}

A total of eighty eyes of eighty consecutive patients (39 men and 41 women) were analyzed. The mean age of the study population was $42.05 \pm 12.51$ years (range 21 to 64 ). The mean spherical equivalent refractive error was $-10.0 \mathrm{D}$ (range -7.0 to $-10.0 \mathrm{D}$ ). There were no statistically significant differences among the two groups in gender, age, refractive error, duration of symptoms and severity of illness (Table 1). 
Table 1. Baseline demographic data in two different groups.

\begin{tabular}{ccc}
\hline & Integrative Group $(\mathrm{n}=40)$ & Control Group $(\mathrm{n}=40)$ \\
\hline Sex (Male/Female) & $21 / 19$ & $18 / 22$ \\
Age, years & $41.33 \pm 12.78$ & $42.78 \pm 12.34$ \\
Refractive error, D & $-10.0 \mathrm{D}$ & $-10.0 \mathrm{D}$ \\
Duration of symptoms, months & $3.02 \pm 1.58$ & $3.56 \pm 1.23$ \\
BCVA, letters & $47.10 \pm 13.51$ & $42.90 \pm 13.18$ \\
CRT, $\mu \mathrm{m}$ & $370.73 \pm 65.31$ & $364.75 \pm 69.31$ \\
\hline
\end{tabular}

\subsection{Change of BCVA}

The evolution of the BCVA data is summarized in Figure 1. After a course of 1-year treatment, patients in both groups had a mean BCVA improvement $(\mathrm{P}<0.05)$. The mean initial BCVA of the integrative group increased after the first month of treatment with a visual gain of 34 letters, and it reached $90.72 \pm 12.98$ letters at the 1-year follow-up. As for the control group, the visual gain was 27 letters, and BCVA reached $69.18 \pm 13.21$ letters at the 1-year follow-up. BCVA of patients in the integrative group improved significantly, more than in the control group since the third month after the initial treatment to the end of the follow-up. The Mann-Whitney test shows significant differences $(\mathrm{P}<0.05)$ between 2 groups at all points of follow-up.

\subsection{Change of CRT}

Patients in both groups underwent a mean CRT improvement $(\mathrm{P}<0.05)$. Mean OCT CRT reduced from $364.75 \pm 69.31$ to $278.93 \pm 33.05$ um in the control group and from $370.73 \pm 65.31$ to $271.73 \pm 46.44 \mathrm{um}$ in the integrative group, respectively. The mean CRT of patients in the integrative group improved significantly, more than in the control group since the third month after the initial treatment to the end of the follow-up. $(\mathrm{P}<0.05)$ (Figure 2).

\subsection{Average Numbers of Intravitreal Injection}

The mean number of intravitreal injections of the patients with cure and effective efficacy was $1.83 \pm 0.34$ in the integrative group and $2.32 \pm 0.57$ in the control group after a course of 1-year treatment, the difference between the two groups was statistically significant $(\mathrm{P}<0.05)$.

\subsection{Safety}

There were no major ocular or systemic problems, such as increased intraocular pressure, retinal detachment, intraocular inflammation, or vascular events during the 1year follow-up in both groups.

\section{Discussion}

The prevalence of myopia varies among ethnic groups with a higher prevalence in 


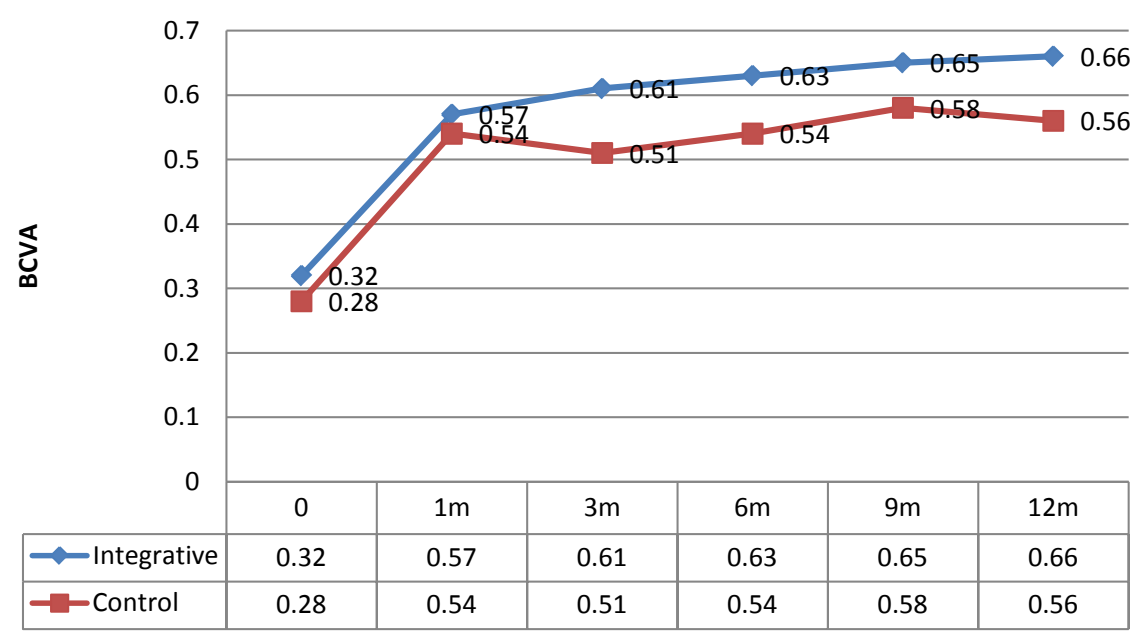

Figure 1. Changes of BCVA in 1-year's treatments and follow-up in two different groups.

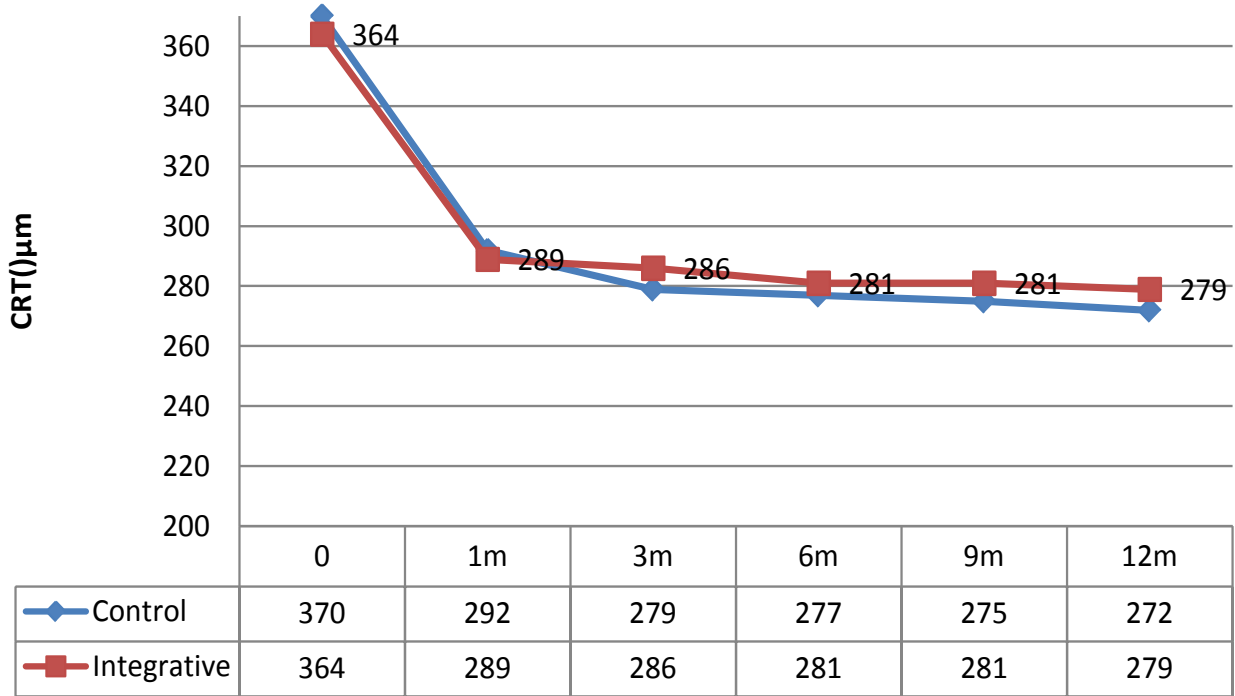

Figure 2. Changes of CRT in 1-year's treatments and follow-up in two different groups.

Asian (17\% - 43\%) compared to white populations (13\% - 27\%) [6]. CNV is the most common vision-threatening complication of pathologic myopia, affecting 5\% - 10\% [7]. As the researchers show mCNV could cause posterior pole myopic retinal changes (lacquer cracks, chorioretinal atrophy, papillary crescent, posterior staphyloma).

Myopic CNV is still a treatment challenge with conventional therapies. Intravitreal injection of anti-VEGF has shown promising results, and is currently considered the first-line therapy for sub-foveal and juxta-foveal mCNV [4]. Ranibizumab (Lucentis; Genentech) is a specific affinity-matured, recombinant, humanized, anti-VEGF antigen-binding antibody fragment that binds and neutralizes all the biologically active forms of VEGF-A. Many publications have shown favorable results of intravitreal ranibizumab for $\mathrm{mCNV}$ [8]. However, repeated intravitreal injections are needed to maintain vision improvement, leading to greater potential risks of complications and ex- 
penses [9].

Our previous studies have shown that combined treatment of traditional Chinese and Western method could have certain advantages [10] [11]. LQHB is a compound Chinese herbal medicine of Shanghai General Hospital (Shanghai medicine system of word: Z05050795). The main components include Angelica sinensis (DangGui), Ligusticum wallichii (ChuanXiong), Salvia miltiorrhiza (DanShen), Atractylodes chinensis (CangZhu), Sargassum pallidum (HaiZao), Cuscuta japonica (TuSiZi), Cistanche deserticola (RouCongRong), Fructus lycii (GouQiZi), etc. The therapeutic effect of LQHB is to strengthen yang, nourish the liver and kidney, enhance virtual supplementation, replenish the blood, and remove obstruction from meridians. Our previous cell experiments as well as animal studies have demonstrated that LQHB could obviously protect both function and morphology of the retina [12] [13]. In clinical applications, LQHB have been used to treat age-related macular degeneration (AMD) patients for over 15 years with great efficacy. Visual capacity is improved by promoting the circulation in choroids and retina, preventing cell death, and reversing the inability to absorb and circulate vital nutrients for healthy vision. This study was designed to demonstrate the therapeutic effect of LQHB for $\mathrm{mCNV}$ when combined with IVR.

In cases of myopic CNV, the bleeding must be stopped first, and then the eyes invigorated. Once the bleeding has been arrested after IVR, yang-strengthening and yinnourishing formulas should be used in raw decoctions. These formulas will be taken long term (3 - 12 months), as it takes a longer amount of time to build the yin than it does to generate yang. The main therapeutic principal of LQHB is promoting the blood circulation, inducing dieresis and nourishing the liver and kidney, while treating mCNV after IVR injection. Myopic CNV is often an exhaustion of the body's yin. The yin-aspect of the visual field is the central vision, and the peripheral vision is more yang in nature. When the central vision goes, it indicated a "burn-out" of kidney and liver yin, so using Chinese herbs that nourish yin seems to work best. Angelica sinensis (DangGui), Ligusticum wallichii (ChuanXiong) and Salvia miltiorrhiza (DanShen) work together to promote the blood circulation, repair the hypoxia damage of tissue and improve the obstruction of exudations; Atractylodes chinensis (CangZhu) and Sargassum pallidum (HaiZao) nourish the spleen, thus inducing dieresis and alleviating macular edema; Cuscuta japonica (TuSiZi), Cistanche deserticola (RouCongRong) and Fructus lycii (GouQiZi) supplement the liver and kidney, build the yin, thus improving visual acuity.

In our paper, we confirm the efficacy of the combined treatment in myopic CNV. The final mean BCVA of the integrative group reached $90.72 \pm 12.98$ letters, with a visual gain of 34 letters when compared with the initial mean BCVA $(\mathrm{P}<0.05)$. As for the control group, the visual gain was 27 letters $(\mathrm{P}<0.05)$. We also found a significant decrease in CRT in both groups $(\mathrm{P}<0.05)$. The mean CRT of patients in the integrative group improved significantly, more than in the control group $(\mathrm{P}<0.05)$. The combined treatment could also lead to lower number of injections, with lower risks and less expense. These findings suggested that LQHB could have significant therapeutic effect in 
treating mCNV when combined with IVR.

\section{Conclusion}

To conclude, LQHB could have significant therapeutic effect in treating mCNV when combined with IVR. Combined therapy appears to be a helpful option for maintaining good vision outcomes, making it possible to reduce the number of doses. Moreover, patients can also benefit from the reduction in risk of complications and the discomfort it entails, while the cost of the process also decreases.

\section{References}

[1] Ohno-Matsui, K., Lai, T.Y., Lai, C.C. and Cheung, C.M. (2016) Updates of Pathologic Myopia. Progress in Retinal and Eye Research, 52, 156-187. http://dx.doi.org/10.1016/j.preteyeres.2015.12.001

[2] Verkicharla, P.K., Ohno-Matsui, K. and Saw, S.M. (2015) Current and Predicted Demographics of High Myopia and an Update of Its Associated Pathological Changes. Ophthalmic and Physiological Optics, 35, 465-475. http://dx.doi.org/10.1111/opo.12238

[3] Teo, K.Y., Ng, W.Y., Lee, S.Y. and Cheung, C.M. (2016) Management of Myopic Choroidal Neovascularization: Focus on Anti-VEGF Therapy. Drugs, 76, 1119-1133. http://dx.doi.org/10.1007/s40265-016-0605-0

[4] Munk, M.R., Rückert, R., Zinkernagel, M., Ebneter, A. and Wolf, S. (2016) The Role of Anti-VEGF Agents in Myopic Choroidal Neovascularization: Current Standards and Future Outlook. Expert Opinion on Biological Therapy, 16, 477-487. http://dx.doi.org/10.1517/14712598.2016.1132696

[5] Zhang, Y., Han, Q., Ru, Y., Bo, Q. and Wei, R.H. (2015) Anti-VEGF Treatment for Myopic Choroid Neovascularization: From Molecular Characterization to Update on Clinical Application. Drug Design, Development and Therapy, 9, 3413-3421. http://dx.doi.org/10.2147/DDDT.S87920

[6] Morgan, I.G., He, M. and Rose, K.A. (2016) Epidemic of Pathologic Myopia: What Can Laboratory Studies and Epidemiology Tell Us? Retina. http://dx.doi.org/10.1097/IAE.0000000000001272

[7] Pakzad-Vaezi, K., Mehta, H., Mammo, Z. and Tufail, A. (2016) Vascular Endothelial Growth Factor Inhibitor Use and Treatment Approach for Choroidal Neovascularization Secondary to Pathologic Myopia. Expert Opinion on Biological Therapy, 16, 873-881. http://dx.doi.org/10.1517/14712598.2016.1167868

[8] Ji, L., Lv, W., Xiao, Y., Xu, Z., Zhang, X. and Zhang, W. (2015) Therapeutic Effect of Intravitreal Injections of Ranibizumab for the Treatment of Macular Choroidal Neovascularization Caused by Pathological Myopia. Experimental and Therapeutic Medicine, 10, 11211126.

[9] Ladaique, M., Dirani, A. and Ambresin, A. (2015) Long-Term Follow-Up of Choroidal Neovascularization in Pathological Myopia Treated with Intravitrealranibizumab. Klin Monbl Augenheilkd, 232, 542-547. http://dx.doi.org/10.1055/s-0035-1545817

[10] Lu, B.W. and Wu, X.W. (2015) Clinical Study of Sulfotanshinone Sodium Injection in Treating Non-Ischemic Retinal Vein Occlusion. Chinese Medicine, 6, 83-89. http://dx.doi.org/10.4236/cm.2015.62009

[11] Lu, B.W. and Wu, X.W. (2015) Retinal Functional Changes Measured by Microperimetry after Intravitreal Ranibizumab Injection and Sulfotanshinone Sodium Injection for Macular 
Edema Secondary to Retinal Vein Occlusion. Chinese Medicine, 6, 181-186.

http://dx.doi.org/10.4236/cm.2015.63020

[12] Gong, Y.Y., Song, Y., Xie, Z.G. and Wu, X.W. (2008) Protective Effect of Huangban Granule against Light-Induced Retinal Damage in Rats. Zhong Xi Yi Jie He Xue Bao, 6, 1159-1163. http://dx.doi.org/10.3736/jcim20081110

[13] Li, C.H., Qiu, Q.H., Wu, X.W., Gong, Y.Y., Xie, Z.G., Song, Y. and Gu, Q. (2012) Chinese Herbal Medicine Lingqi Huangban Granule Protects Retinal Pigment Epithelial Cells Against Oxidative Stress-Induced Injury in Vitro. Zhong Xi Yi Jie He Xue Bao, 10, 85-90. http://dx.doi.org/10.3736/jcim20120113

\section{Submit or recommend next manuscript to SCIRP and we will provide best service} for you:

Accepting pre-submission inquiries through Email, Facebook, LinkedIn, Twitter, etc. A wide selection of journals (inclusive of 9 subjects, more than 200 journals)

Providing 24-hour high-quality service

User-friendly online submission system

Fair and swift peer-review system

Efficient typesetting and proofreading procedure

Display of the result of downloads and visits, as well as the number of cited articles

Maximum dissemination of your research work

Submit your manuscript at: http://papersubmission.scirp.org/

Or contact cm@scirp.org 